\title{
Levantamento da fauna de Triatominae (Hemiptera: Reduviidae) em ambiente domiciliar e infecção natural por Trypanosomatidae no Estado de Mato Grosso do Sul
}

\author{
Survey of Triatominae (Hemiptera: Reduviidae) fauna in domestic \\ environments and natural infection by Trypanosomatidae \\ in the State of Mato Grosso do Sul
}

\author{
Paulo Silva de Almeida ${ }^{1,2}$, Walter Ceretti Júnior ${ }^{3}$, Marcos Takashi Obara ${ }^{3}$, \\ Honório Roberto Santos ${ }^{2}$, José Maria Soares Barata ${ }^{3}$ e Odival Faccenda ${ }^{4}$
}

\begin{abstract}
RESUMO
Entre 2000 a 2004, foi realizado levantamento da fauna de Triatominae (Hemiptera: Reduviidae) e exame de infecção natural por Trypanosomatidae, no Estado de Mato Grosso do Sul. Um total de 13.671 espécimes foram capturados. Na análise faunística das espécies capturadas, Triatoma sordida foi caracterizada como muito abundante, muito frequiente, constante e dominante. Os índices de infecção natural para Trypanosoma cruzi apresentaram os valores de 3,2\% para Panstrongylus geniculatus, 0,6\% para Rhodnius neglectus e 0,1\% para Triatoma sordida, apesar do Estado de Mato Grosso do Sul apresentar-se livre da transmissão vetorial endêmica.
\end{abstract}

Palavras-chaves: Doença de Chagas. Intradomicílio. Peridomicílio. Riqueza de espécies.

\begin{abstract}
Between 2000 and 2004, a survey of Triatominae fauna (Hemiptera: Reduviidae) and examination of natural infection caused by Trypanosomatidae in the State of Mato Grosso do Sul, Brazil, was conducted. A total of 13,671 specimens were collected. Through fauna analysis on the insects that were caught, Triatoma sordida was characterized as very abundant, very frequent, constant and dominant. The rates of natural infection with Trypanosoma cruzi were $3.2 \%$ for Panstrongylus geniculatus, $0.6 \%$ for Rhodnius neglectus and $0.1 \%$ for Triatoma sordida. Nevertheless, the State of Mato Grosso do Sul is free from endemic vector transmission.
\end{abstract}

Key-words: Chagas disease. Intradomicile. Peridomicile. Species richness.

Atualmente são conhecidas 138 espécies de triatomíneos agrupadas em 6 tribos formadas por 19 gêneros. Entretanto, somente algumas são vetores efetivos da doença de Chagas, devido à adaptação aos ambientes domésticos e a antropofilia ${ }^{3811}$. Dentre essas espécies, destacam-se: Rhodnius prolixus (Stal, 1859), Triatoma infestans (Klug, 1834), Triatoma dimidiata (Letreille, 1811), Triatoma brasiliensis (Neiva, 1911) e Panstrongylus megistus (Burmeister, 1835) como os principais vetores de Trypanosoma cruzi (Chagas, 1909) na América Latina ${ }^{16}$.

No Brasil, as cinco espécies mais importantes na transmissão do agente causador da doença de Chagas ao homem são: Triatoma infestans, espécie introduzida, que em relação às demais apresenta, maior antropofilia e que historicamente, alcançou ampla distribuição nos estados do sul, sudeste, centrooeste e nordeste. Em 2006, o Brasil recebeu a Certificação pela Interrupção da Doença de Chagas pelo Triatoma infestans estando a espécie controlada em níveis que não sustentam a transmissão do agente etiológico da doença; Panstrongylus megistus, espécie nativa e domiciliada em extensa área das regiões litorâneas do nordeste e sudeste; Triatoma brasiliensis, de larga distribuição no semi-árido do nordeste; Triatoma pseudomaculata (Corrêa \& Espínola, 1964), ocorrendo também no semi-árido do nordeste e Triatoma sordida (Stal, 1859), espécie nativa do cerrado ${ }^{1822} 26$.

\footnotetext{
1. Laboratório Regional de Entomologia, Núcleo Regional de Saúde, Secretaria de Estado de Saúde, Dourados, MS. 2. Departamento de Ciências Biológicas, Universidade Federal da Grande Dourados, Dourados, MS. 3. Departamento de Epidemiologia, Faculdade de Saúde Pública, Universidade de São Paulo, São Paulo, SP. 4. Universidade Estadual de Mato Grosso do Sul, Dourados, MS.

Endereço para correspondência: Dr. Paulo Silva de Almeida. Laboratório Regional de Saúde/NRS/SES. Rua Hilda Bergo Duarte, 940, Centro, 79806-020, Centro, Dourados, MS. Tel: 5567 3421-4672; Fax: 5567 3421-4111

e-mail: psilvadealmeida@yahoo.com.br

Recebido em 10/09/2007

Aceito em 07/07/2008
} 
Triatoma sordida por ser nativa, pode reinvadir as habitações a partir dos ecótopos silvestres. Essa espécie, predominante nos cerrados, sobretudo da região Centro-oeste, apresenta ainda marcada ornitofilia e está presente em ninhos de aves, madeira seca ou, ecótopos artificiais, como galinheiros, quando a oferta alimentar está esgotada nesses hábitats, podendo invadir e colonizar as casas ${ }^{621}$.

Os triatomíneos desenvolvem a capacidade de domiciliação quando ocorre a alteração da cobertura vegetal primitiva provocada pela ação antrópica, associada às péssimas condições sócio-econômicas e as precárias moradias do homem do campo resultando na biocenose Trypanosoma cruzi-triatomíneohomem, isto é: mal de Chagas?.

As condições necessárias para ocorrer a transmissão domiciliar da doença de Chagas dependem, ainda, de variáveis ou atributos de cada espécie de vetor, como a capacidade de colonização e o tamanho da colônia no interior da casa, ou seja, da densidade populacional e de sua capacidade vetorial de se infectar e transmitir o Trypanosoma cruzi ao homem ${ }^{1820}$.

Silveira e cols ${ }^{21}$ realizaram pesquisas domiciliares de triatomíneos em 50 municípios do Estado de Mato Grosso do Sul; destes, 38 detectaram a presença de Triatoma infestans, representando 76\% dos municípios investigados.

As atividades para o controle do vetor da doença de Chagas em Mato Grosso do Sul foram implementados a partir de 1992, quando foi implantado do Programa de Eliminação do Triatoma infestans (PETi). Através de levantamentos realizados em localidades com história da presença desta espécie, constatou-se o registro da mesma em 23 municípios do estado (CORE/MS) ${ }^{13}$.

A história da presença do Triatoma infestans nesse estado, iniciou-se nas décadas de 70 e 80, sempre apresentando baixa densidade e, geralmente, no ambiente intradomiciliar, caracterizandose como espécie de introdução recente e passiva, provavelmente relacionada a expansão das atividades produtivas dessa região.

Com a expansão das atividades do Programa de Controle da Doença de Chagas no Estado de Mato Grosso do Sul, principalmente na década de 80 , esse triatomíneo praticamente foi controlado em quase toda a região, sendo apenas registrado em 3 municípios (Bela Vista em 1991, Dourados em 1992 e Amambai em 1995), sempre em baixa densidade. Após a conclusão das ações de controle da doença de Chagas no Estado de Mato Grosso do Sul em 1995, a Organização Pan-americana de Saúde (OPAS), certificou a eliminação da espécie nos domicílios ${ }^{15}$.

Nesta pesquisa, procurou-se conhecer a fauna e abundância das espécies de triatomíneos em ambiente domiciliar, assim como determinar o índice de infecção natural por flagelados (Trypanosomatidae) no Estado de Mato Grosso do Sul, visando fornecer subsídios para realizar o controle efetivo desses vetores em áreas de alta densidade populacional.

\section{MATERIAL E MÉTODOS}

0 Estado de Mato Grosso do Sul possui 77 municípios, distribuídos em uma área de $358.168 \mathrm{~km}^{2}$ com população estimada de 2.264.468 habitantes (IBGE) ${ }^{10}$. Limita-se a leste com os Estados de Minas Gerais e São Paulo, ao norte com os estados de Mato Grosso e Goiás, ao sul com Paraná e Paraguai e ao oeste com a Bolívia.

O estado atualmente está dividido em cinco Núcleos Técnicos de Vetores, conforme estabelecido pela Coordenadoria de Controle de Vetores (CCV) da Secretaria Estadual de Saúde/SES (Figura 1).

Para conhecer a fauna triatomínica do Estado de Mato Grosso do Sul, analisaram-se dados secundários em bancos que contêm as informações trabalhadas no Estado de Mato Grosso do Sul no Controle da Doença de Chagas (PCDCh) desenvolvido pela Coordenadoria de Controle de Vetores no período de 2000 a 2004. As ações de vigilância preconizaram atividades de pesquisa integral de captura de triatomíneos (rotina) e atendimento de notificação pela população por meio de Postos de Informação de Triatomíneos (PITs) em cinco Núcleos Técnicos de Vetores (56 municípios) do estado. A periodicidade da pesquisa de rotina no PCDCh é habitualmente bienal. Consideraram-se os índices obtidos em atividades de rotina: Índice de Dispersão: $\mathrm{n}^{\circ}$ localidades positivas $/ \mathrm{n}^{\mathrm{0}}$ localidades pesquisadas X 100 que determina a infestação com referência a localidades em um município; Índice de Infestação Domiciliar: $\mathrm{n}^{0}$ de UD positivas/nํㅡㄴ UD pesquisadas X 100 este determina a localidade a ser trabalhada para o biênio seguinte e Índice de Infecção Natural: $\mathrm{n}^{\circ}$ de triatomíneos infectados por Trypanosoma cruzi $/ \mathrm{n}^{0} \mathrm{de}$ triatomíneos examinados X 100, em exame laboratorial de conteúdo intestinal de triatomíneos, em suspensão salina, em aumento de 100 a 400 vezes $^{14}$.

No laboratório, os espécimes foram identificados por meio de chaves dicotômicas ${ }^{311}$. A pesquisa de infecção natural pelo Trypanosoma cruzi foi realizada, mediante dissecação dos triatomíneos. Procederam-se exames a fresco e coloração das lâminas pelo método Giemsa ${ }^{23}$.

Foram determinadas as distribuições anuais das espécies de triatomíneos e, posteriormente, realizou-se a análise faunística para definir as classes de abundância, constância e dominância.

0 cálculo de abundância foi determinado pela soma total dos indivíduos de cada espécie, empregando-se uma medida de dispersão, conforme Thomazini \& Thomazini ${ }^{25}$, por meio do cálculo do desvio padrão da média aritmética a um intervalo de confiança (IC), verificado pelo teste t, a 1 e $5 \%$ de probabilidade. Foram estabelecidas as seguintes classes de abundância: $\mathrm{ma}=$ muito abundante (número de indivíduos maior que o limite superior do IC a 1\%); a = abundante (número de indivíduos situado entre os limites superiores do IC a 5 e a 1\%); $\mathrm{c}=$ comum (número de indivíduos situado dentro do IC a 5\%); $\mathrm{d}$ = dispersa (número de indivíduos situado entre os limites inferiores do IC a 5 e a 1\%) e r = rara (número de indivíduos menor que o limite inferior do IC a 1\%).

0 intervalo de confiança da média das freqüências (porcentagem de indivíduos coletados) com $5 \%$ de probabilidade 


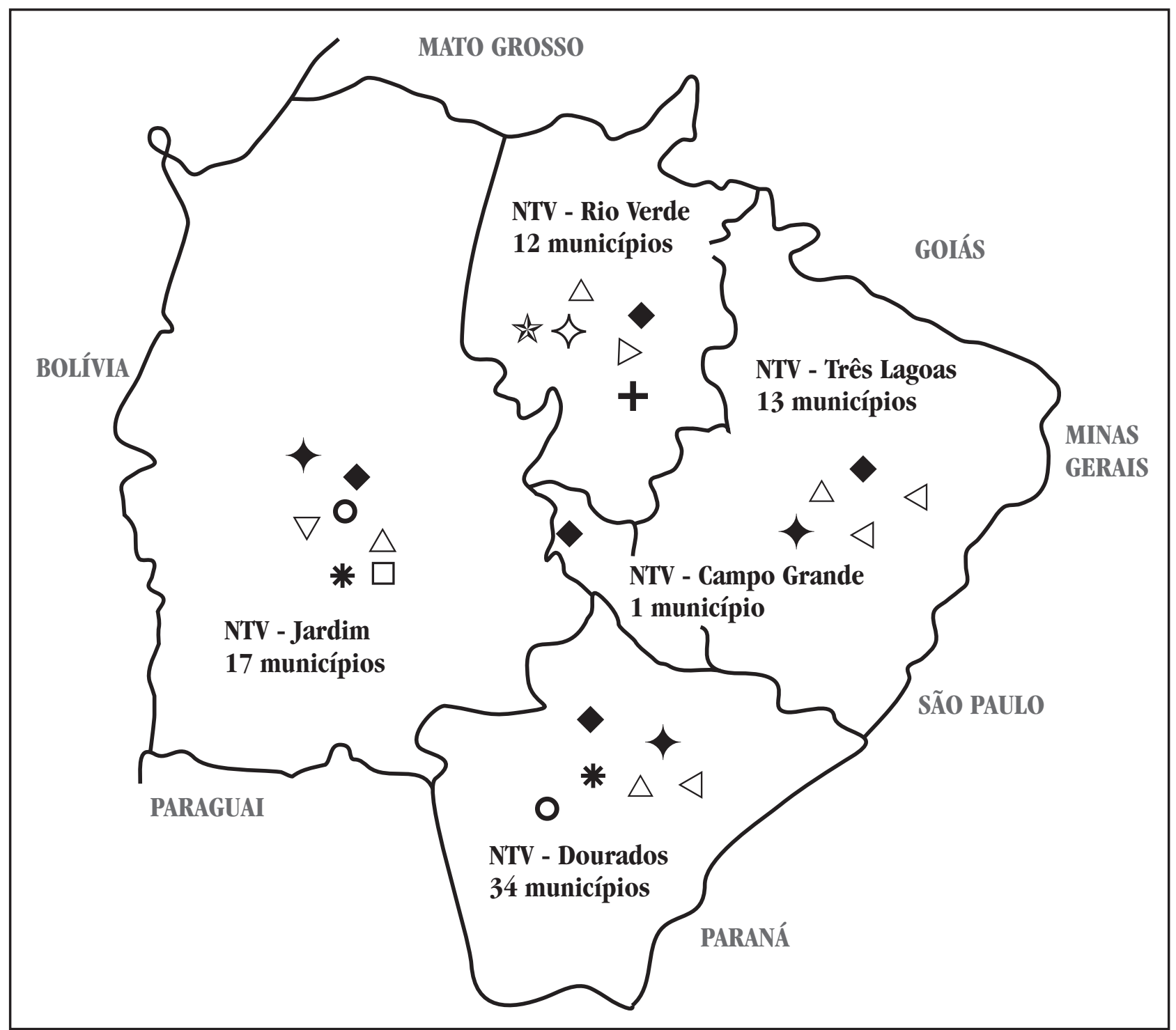

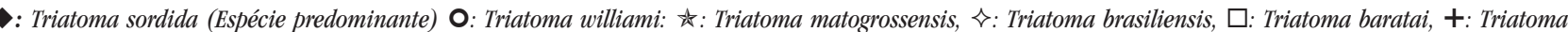
vandae, $\triangle$ :Rhodnius neglectus, $\nabla$ :Rhodnius pictipes, $\triangleleft$ : Panstrongylus diasi, $\triangleright:$ : Panstrongylus geniculatus: * Panstrongylus guentheri, $\uparrow:$ Panstrongylus megistus. NTV: Núcleo Técnico de Vetores.

Figura 1 - Distribuição das espécies de triatomíneos capturados nos Núcleos Técnicos de Vetores da Coordenadoria de Controle de Vetores, no Estado de Mato Grosso do Sul, período de 2000 a 2004.

foi determinado pela seguinte classificação: $\mathrm{mf}=$ muito freqüente (frequiência menor que o limite superior do IC a 5\%); $\mathrm{f}=$ frequiente (frequiência situada dentro do IC a $5 \%$ ) e pf = pouco freqüente (frequiência menor que o limite inferior do IC a 1\%).

A constância foi avaliada por meio da porcentagem de coletas que continham uma determinada espécie, usando-se a fórmula: $\mathrm{C}=$ (número de coletas com espécie $\mathrm{X} /$ número total de coletas) $x$ 100. De acordo com os valores obtidos as espécies foram classificadas em: $\mathrm{w}=$ constante $(\mathrm{C}>50 \%) ; \mathrm{y}=$ acessória (C entre 25 e $50 \%)$ e $\mathrm{z}=$ acidental $(\mathrm{C}<25 \%)$.

As espécies predominantes foram aquelas que se destacaram por obter os maiores índices faunísticos de abundância, frequiência, constância e dominância ${ }^{24}$.

Para verificar qual a espécie de triatomíneo está associada a determinado Núcleo Técnico de Vetores, utilizou-se o método de análise de correspondência ${ }^{12}$.

\section{RESULTADOS}

Durante o período trabalhado, foram coletados 13.671 exemplares de triatomíneos, onde $12,4 \%$ estavam presentes no intradomicílio e 87,6\% no peridomicílio. Triatoma sordida foi à espécie mais abundante com 13.102 exemplares, correspondendo a 95,8\% do total de insetos coletados; seguida por Rhodnius neglectus (Lent, 1954) (415 exemplares), Panstrongylus geniculatus (Latreille, 1291) (51 exemplares) e Triatoma williami (Galvão et al 1965) (29 exemplares). As demais espécies capturadas: Panstrongylus megistus, Triatoma baratai (Carcavallo \& Jurberg, 2000), Triatoma brasiliensis, (Neiva, 1911) Triatoma matogrossensis (Leite \& Barbosa, 1953), Triatoma vandae (Carcavallo et al 2002), Rhodnius pictipes (Stal, 1872), Panstrongylus diasi (Pinto \& Lent, 1946) e Panstrongylus guentheri (Berg, 1879), juntas perfizeram apenas 74 exemplares dos insetos capturados (Tabela 1). 
Tabela 1 - Frequiência das principais espécies de triatomíneos capturados (intra e peridomicílio) no Estado de Mato Grosso do Sul, no período de 2000 a 2004.

\begin{tabular}{|c|c|c|c|c|}
\hline \multirow[b]{2}{*}{ Espécies } & \multicolumn{3}{|c|}{ Local de captura (\%) } & \multirow[b]{2}{*}{ Total (\%) } \\
\hline & capturados $\left(\mathrm{n}^{0}\right)$ & intradomicílio (\%) & peridomicílio (\%) & \\
\hline Panstrongylus diasi & 2 & 0,0 & 0,0 & 0,0 \\
\hline Panstrongylus geniculatus & 51 & 0,2 & 0,1 & 0,4 \\
\hline Panstrongylus guentheri & 2 & 0,0 & 0,0 & 0,0 \\
\hline Panstrongylus megistus & 19 & 0,1 & 0,0 & 0,1 \\
\hline Rhodnius neglectus & 415 & 2,4 & 0,6 & 3,0 \\
\hline Rhodnius pictipes & 2 & 0,0 & 0,0 & 0,0 \\
\hline Triatoma matogrossensis & 19 & 0,0 & 0,1 & 0,1 \\
\hline Triatoma brasiliensis & 3 & 0,0 & 0,0 & 0,0 \\
\hline Triatoma baratai & 22 & 0,1 & 0,0 & 0,2 \\
\hline Triatoma sordida & 13.102 & 9,3 & 86,6 & 95,8 \\
\hline Triatoma vandae & 5 & 0,0 & 0,0 & 0,0 \\
\hline Triatoma williami & 29 & 0,1 & 0,1 & 0,2 \\
\hline$\overline{\text { Total }}$ & 13.671 & 12,4 & 87,6 & 100,0 \\
\hline
\end{tabular}

Por meio da análise dos parâmetros faunísticos realizados para as espécies de triatomíneos, Triatoma sordida, foi caracterizada como muito abundante, enquanto as espécies restantes foram caracterizadas como comuns.

Na avaliação da constância, o número de vezes que se recuperou uma determinada espécie no total de coletas efetuadas, observou-se que as espécies que estiveram mais presente foram Triatoma sordida, Triatoma williami, Triatoma brasiliensis, Rhodnius negletus, Panstrongylus megistus, Panstrongylus geniculatus, foram caracterizadas como constantes. Apenas Triatoma matogrossensis foi considerada como acessória. As espécies Panstrongylus diasi, Panstrongylus guentheri, Rhodnius pictipes, Triatoma baratai e Triatoma wandae foram caracterizadas como não constantes.

Em relação ao índice faunístico de frequiência para o Estado de Mato Grosso do Sul, a espécie Triatoma sordida foi caracterizada como muito freqüente. A espécie Rhodnius neglectus foi espécie freqüente, enquanto as demais foram caracterizadas como pouco freqüente. Triatoma sordida foi caracterizada espécie dominante na maioria dos municípios e todas as demais espécies capturadas foram classificadas como não dominantes (Tabela 2).

Com relação ao índice de infecção natural por Trypanosoma cruzi o maior índice obtido refere-se a Panstrongylus geniculatus (3,2\%), seguido por Rhodnius neglectus (0,6\%) e Triatoma sordida $(0,1 \%)$. Não foram observados indivíduos com infecção natural dentre as demais espécies capturadas(Tabela 3).

Foi observada uma relação significativa $\left(\mathrm{X}^{2}=341,32\right.$; $\mathrm{p} \leq 0,01$ ) entre a abundância das espécies e o local de captura (Tabela 4). 0 peridomicílio sempre apresentou maior abundância do que o intradomicílio. 0 Núcleo Técnico de Vetores de Três Lagoas apresentou o maior destaque com $29 \%$ dos triatomíneos capturados neste ambiente, seguido dos Núcleos de Jardim com $27 \%$ e Rio Verde com 20\%. Os Núcleos Técnicos de Vetores de Campo Grande (5\%) e Dourados (8\%) apresentaram as menores abundâncias de indivíduos capturados (Tabela 4).

Tabela 2 - Espécies de triatomíneos capturados em 56 municípios do Estado de Mato Grosso do Sul e suas respectivas classes de Abundância (A), Constância (C), Frequiência (F) e Dominância (D), no período de 2000 a 2004.

\begin{tabular}{|c|c|c|c|c|c|}
\hline Espécies & Capturados $^{1}$ & Abundância ${ }^{2}$ & Constância ${ }^{3}$ & Frequiência ${ }^{4}$ & Dominância \\
\hline Panstrongylus diasi & 2 & $\mathrm{c}$ & $\mathrm{Z}$ & Pf & $\mathrm{n}$ \\
\hline Panstrongylus geniculatus & 51 & $\mathrm{c}$ & w & Pf & $\mathrm{n}$ \\
\hline Panstrongylus guentheri & 2 & $\mathrm{c}$ & Z & Pf & $\mathrm{n}$ \\
\hline Panstrongylus megistus & 19 & c & w & Pf & $\mathrm{n}$ \\
\hline Rhodnius neglectus & 415 & $\mathrm{c}$ & w & $\mathrm{F}$ & $\mathrm{n}$ \\
\hline Rhodnius pictipes & 2 & $\mathrm{c}$ & $\mathrm{z}$ & Pf & $\mathrm{n}$ \\
\hline Triatoma matogrossensis & 19 & $\mathrm{c}$ & $\mathrm{y}$ & Pf & $\mathrm{n}$ \\
\hline Triatoma brasiliensis & 3 & $\mathrm{c}$ & w & Pf & $\mathrm{n}$ \\
\hline Triatoma baratai & 22 & $\mathrm{c}$ & $\mathrm{z}$ & Pf & $\mathrm{n}$ \\
\hline Triatoma sordida & 13.102 & $\mathrm{ma}$ & w & Mf & $\mathrm{s}$ \\
\hline Triatoma vandae & 5 & $\mathrm{c}$ & $\mathrm{z}$ & Pf & $\mathrm{n}$ \\
\hline Triatoma williami & 29 & $\mathrm{c}$ & w & Pf & $\mathrm{n}$ \\
\hline Total & 13.671 & & & & \\
\hline
\end{tabular}

A: Abundância, ma: muito abundante, a: abundante, c: comum, d: dispersa.

F: Freqüência, mf: muito freqüente, f: freqüente, pf: pouco freqüente.

C: Constância, w: constante, y: acessória, z: acidental.

D: Dominância, s: dominante, n: não dominante. 
Tabela 3. Distribuição das espécies de triatomíneos examinados e índice de infecção natural por Trypanosomatidae no Estado de Mato Grosso do Sul, no período de 2000 a 2004.

\begin{tabular}{lccc}
\hline Espécies & Examinados & Infectados & Infectados (\%) \\
\hline Panstrongylus diasi & 0 & 0 & 0,0 \\
Panstrongylus geniculatus & 31 & 1 & 3,2 \\
Panstrongylus guentheri & 0 & 0 & 0,0 \\
Panstrongylus megistus & 13 & 1 & 0,0 \\
Rhodnius neglectus & 154 & 0 & 0,6 \\
Rhodnius pictipes & 0 & 0 & 0,0 \\
Triatoma matogrossensis & 19 & 0 & 0,0 \\
Triatoma brasiliensis & 2 & 0 & 0,0 \\
Triatoma baratai & 10 & 0 & 0,0 \\
Triatoma sordida & 8.615 & 13 & 0,2 \\
Triatoma vandae & 0 & 0 & 0,0 \\
Triatoma williami & 24 & 0 & 0,0 \\
\hline Total & $\mathbf{8 . 8 6 8}$ & $\mathbf{1 5}$ & $\boldsymbol{0 , 2}$ \\
\hline
\end{tabular}

Tabela 4 - Distribuição dos triatomíneos capturados no (intra e peridomicílio) e índice de infecção natural por Trypanasomatidae nos Núcleos Técnicos de Vetores do Estado de Mato Grosso do Sul, no período de 2000 a 2004.

\begin{tabular}{|c|c|c|c|c|c|}
\hline \multirow[b]{3}{*}{ Núcleos } & \multirow[b]{3}{*}{ Indivíduos } & \multicolumn{4}{|c|}{ Local de capturas e infectados } \\
\hline & & \multicolumn{2}{|c|}{ intradomicílio } & \multicolumn{2}{|c|}{ peridomicílio } \\
\hline & & capturados & $\overline{\text { infectados }}$ & capturados & infectados \\
\hline Técnicos & capturados & $\%$ & $\%$ & $\%$ & $\%$ \\
\hline Campo Grande & 628 & 0,0 & 0,0 & 5,0 & 0,0 \\
\hline Dourados & 1.321 & 2,0 & 1,2 & 8,0 & 0,0 \\
\hline Jardim & 3.981 & 2,0 & 0,7 & 27,0 & 0,0 \\
\hline Rio Verde & 3.012 & 2,0 & 0,5 & 20,0 & 0,0 \\
\hline Três Lagoas & 4.729 & 5,0 & 0,3 & 29,0 & 0,1 \\
\hline Total & 13.671 & 11,0 & 0,5 & 89,0 & 0,1 \\
\hline
\end{tabular}

$\chi^{2}=341,32 ;(\mathrm{P}<0,01)$

Constatou-se uma diferença significativa do índice de infecção natural por Trypanosoma cruzi entre os Núcleos Técnicos de Vetores e o local de captura intradomicílio e peridomicílio. 0 intradomícilio apresentou maior índice de infecção natural de $0,5 \%$ e o peridomicílio registrou o índice de apenas $0,1 \%$. O Núcleo Técnico de Vetores de Dourados apresentou maior índice de infecção natural de Trypanosoma cruzi de 1,2\% no intradomicílio, seguida do Núcleo de Jardim com 0,7\%, Núcleo de Rio Verde com 0,5\% e o Núcleo de Três Lagoas com 0,3\%. Em relação ao peridomicílio, somente o Núcleo de Três Lagoas que observou o índice de infecção natural por Trypanosoma cruzi de $0,1 \%$ (Tabela 4 ).

A espécie predominante no Estado de Mato Grosso do Sul, Triatoma sordida, esteve presente nos cinco Núcleos Técnicos de Vetores estudados. Rhodnius neglectus, ausente apenas no Núcleo Técnico de Vetores de Campo Grande, também se destacou pela ampla distribuição. Merecem atenção ainda, as espécies: Panstrongylus geniculatus, presente nos Núcleos Técnicos de Vetores de Dourados, Rio Verde e Três Lagoas e Panstrongylus megistus, nos Núcleos Técnicos de Vetores de Dourados, Rio Verde e Jardim. As espécies restantes estiveram apenas em um Núcleo Técnico de Vetores apresentando distribuição geográfica mais restrita (Figura 1).

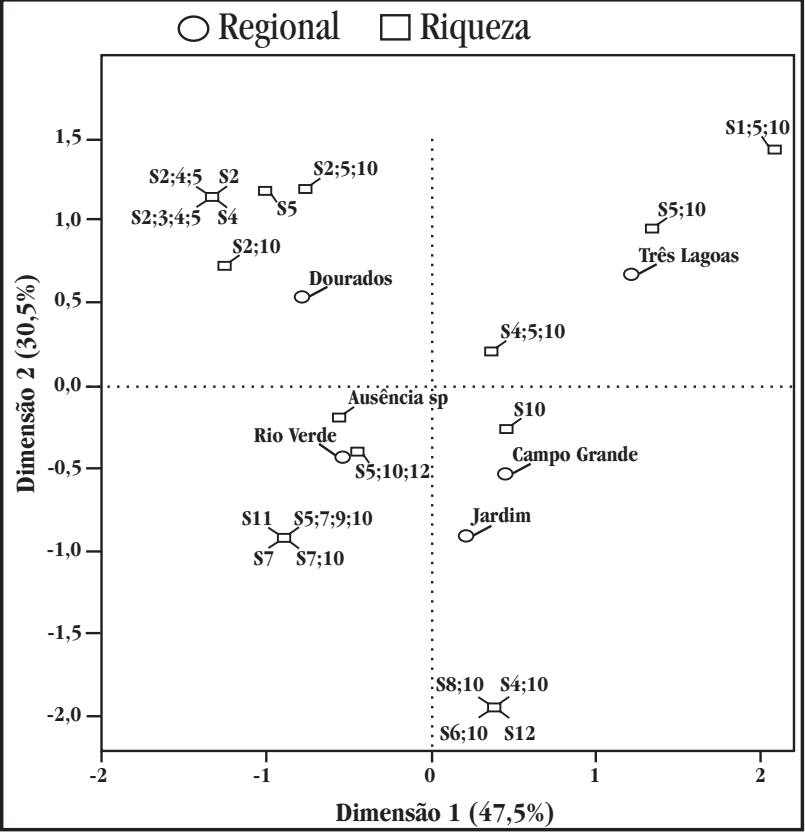

S1: Panstrongilus diasi, S2: Panstrongilus geniculatus, S3: Panstrongilus guentheri, S4: Panstrongilus megistus, S5: Rhodnius neglectus, S6: Rhodnius pictipes, S7: Triatoma matogrossensis, S8: Triatoma baratai, S9: Triatoma brasiliensis, S10: Triatoma sordida, S11: Triatoma vandae, S12: Triatoma williami.

Figura 2 - Análise de correspondência entre as variáveis: Núcleos Técnicos de Vetores e infestação por espécies de triatomíneos capturados no Estado de Mato Grosso do Sul, no período de 2000 a 2004.

Mediante a análise de correspondência foi possível associar as espécies de triatomíneos com os Núcleos Técnico de Vetores do Estado de Mato Grosso do Sul (Figura 2).

O Núcleo Técnico de Dourados apresenta municípios associados com a infestação pelas espécies: Panstrongylus guentheri, Panstrongylus geniculatus, Panstrongylus megistus e Rhodnius neglectus. Existem municípios com infestação por Panstrongylus geniculatus, Rhodnius neglectus e Triatoma sordida e, ainda, por duas espécies: Panstrongylus geniculatus e Triatoma sordida. Quanto ao Núcleo Técnico de Vetores de Três Lagoas, este foi associada a municípios com infestação por três espécies: Panstrongylus diasi, Rhodnius neglectus e Triatoma sordida e duas espécies: Rhodnius neglectus e Triatoma sordida.

Com exceção de Triatoma sordida, com ampla predominância em todos os Núcleos Técnicos de Vetores, nos Núcleos Técnicos de Vetores de Campo Grande, Jardim e Rio Verde, geograficamente muito próximas, não houve clara discriminação da riqueza de espécie entre os municípios. Porém as espécies Rhodnius neglectus Triatoma brasiliensis, Triatoma williami, Triatoma matogrossensis, Triatoma vandae, Triatoma baratai e Rhodnius pictipes também se destacaram.

Os Núcleos Técnicos de Vetores de Dourados e Rio Verde apresentaram maior riqueza de espécies de triatomíneos, enquanto os Núcleos Técnicos de Vetores de Campo Grande e Três Lagoas nos quais esses insetos foram ausentes.

Das 6.602 localidades pesquisadas nos Núcleos Técnicos de Vetores do Estado de Mato Grosso do Sul no período de 2.000 a 2.004 , cerca de 2.120 estavam positivas por triatomíneos, 
enquanto a média da taxa de dispersão foi 34\% entre os Núcleos pesquisados. Durante o período de estudo foram pesquisadas 49.626 unidades domiciliares, destas 3.960 estavam positivas com triatomíneos e a média do índice de infestação entre os Núcleos Técnicos de Vetores de 12\% (Tabela 5).

Tabela 5 - Número de localidades e unidades domiciliares pesquisadas nos Núcleos Técnicos de Vetores do Estado de Mato Grosso do Sul, no período de 2000 a 2004.

\begin{tabular}{lccccccc}
\hline Núcleos & \multicolumn{3}{c}{ Localidades } & & \multicolumn{3}{c}{ Unidades domiciliares } \\
\cline { 2 - 4 } \cline { 7 - 8 } Regionais & pesquisadas & positivas & $\begin{array}{c}\text { dispersão } \\
\text { nesquisadas }\end{array}$ & positivas & índice \\
Campo Grande & 986 & 146 & 13,8 & & 6.301 & 207 & 2,0 \\
Dourados & 681 & 192 & 29,7 & & 10.526 & 218 & 2,3 \\
Jardim & 1.708 & 580 & 35,9 & & 18.691 & 1.519 & 6,7 \\
Rio Verde & 1.189 & 336 & 42,0 & & 4.249 & 566 & 32,5 \\
Três Lagoas & 2.038 & 866 & 48,7 & & 9.859 & 1.450 & 16,6 \\
\hline Total & $\mathbf{6 . 6 0 2}$ & $\mathbf{2 . 1 2 0}$ & $\mathbf{3 4 , 0}$ & $\mathbf{4 9 . 6 2 6}$ & $\mathbf{3 . 9 6 0}$ & $\mathbf{1 2 , 0}$ \\
\hline
\end{tabular}

\section{DISCUSSÃO}

Os resultados obtidos neste trabalho evidenciam um aumento da diversidade de espécies de triatomíneos relacionadas com o ambiente antrópico no Estado de Mato Grosso do Sul, uma vez que em levantamento anterior foram investigados 50 municípios e capturadas nove espécies no estado ${ }^{21}$. Neste trabalho foram investigados 56 municípios do Estado e obtevese um total de 12 espécies. Ressalta-se a captura de espécies, até então, consideradas como estritamente silvestres, ocupando principalmente o peridomicílio, tais como Triatoma vandae e Triatoma baratai que foram descritas recentemente e pouco se sabe sobre seu comportamento, biologia e potencial como vetores do mal de Chagas.

Ainda em relação às espécies silvestres, destaca-se também o encontro de Panstrongylus diasi, detectado na região de Santa Cruz (Bolívia) com dispersão que segue a progressão da espécie pelos Estados mais oriental (MG, SP, G0), provavelmente já estando em Mato Grosso do Sul há muitos anos ${ }^{3}$. A espécie Panstrongylus guentheri, havia sido observada somente na Argentina, Paraguai e Bolívia, cuja ocorrência no Brasil foi detectada recentemente por Almeida cols ${ }^{1}$.

Triatoma sordida foi a espécie mais abundante, com alta predominância e ampla distribuição geográfica no Estado de Mato Grosso do Sul, tal espécie tem a tendência de dispersar-se mais e, sobretudo, colonizar-se no intradomicílio pontos esses que indicariam uma tendência ao aumento de risco de transmissão da protozoose. A ação antrópica e a ocupação do solo (produção, demografia) em Mato Grosso do Sul estão urbanizando e aumentado a sobremaneira os cultivos extensivos de grãos, ao lado da pecuária extensiva. Esses resultados corroboram aos obtidos por Diotaiuti ${ }^{6}$, Ministério da Saúde ${ }^{13}$ e Silveira ${ }^{1921}$. Para esses autores, após a eliminação das populações domiciliadas de Triatoma infestans, essa espécie foi capturada em maior abundância nesse estado e está presente principalmente nos ecótopos peridomiciliares.
Aqui cabe comentar, a presença de Rhodnius neglectus em quatro Núcleos Técnicos de Vetores e de Panstrongylus geniculatus e Panstrongylus megistus que estiveram presentes em três Núcleos Técnicos de Vetores.

Rhodnius neglectus foi a segunda espécie mais capturada e apresentou um índice de infecção maior que Triatoma sordida. É uma espécie silvestre que está associada a palmeiras, porém, pode invadir ecótopos artificiais, atraídos provavelmente pela luz, quando ocorre desequilíbrio ecológico decorrente do desmatamento do ambiente natural, seguida pela diminuição da fonte alimentar ${ }^{2}$. Na região norte de MS, o aparecimento de Rhodnius neglectus deve estar relacionado com os buritis e na região central com os bacuris. Rhodnius pictipes também é basicamente uma espécie de hábitos silvestres, em geral, associada a palmeiras (Scheela spp) e bromeliáceas (Aechmea spp). No entanto, há registros de seu encontro em habitações humanas, possivelmente relacionados a atração desta espécie por luz artificial. Essa espécie tem sido encontrada naturalmente infectada por Trypanosoma cruzi ${ }^{11}$.

Quanto a presença de Pantrongylus megistus no Estado, tal aspecto parece ser relacionado com a redução da cobertura vegetal e sua domiciliação provavelmente é conseqüência da ação antrópica dessa paisagem, cada vez mais a espécie sendo confinada em resíduos de mata, a medida em que avançam os desmatamentos e cultivos extensivos ${ }^{7}$.

0 índice de infecção natural por Trypanosoma cruzi demonstrados neste trabalho, foram baixos, concordando com os índices anteriores verificados pelo Ministério da Saúde ${ }^{15}$, Silveira ${ }^{19}$ e Dias cols ${ }^{4}$, os quais obtiveram 1,2\% de índice de infecção para Triatoma sordida no Estado de Minas Gerais.

Os dados deste trabalho apontam para uma alta infestação por Triatoma sordida relacionada ao peridomicílio. Os baixos índices de infecção natural para o Trypanosoma cruzi não só desta espécie, mas também de outras que estiveram presentes durante o período de estudo, demonstram que a transmissão vetorial da doença de Chagas no Estado de Mato Grosso do Sul está controlada, uma vez que se constatou a eliminação do Triatoma infestans do território desse estado, como ocorreu em São Paulo e Lassance em Minas Gerais ${ }^{517}$.

Destacamos que o Programa de Controle da Doença de Chagas (PCDCh) no Estado de Mato Grosso do Sul está direcionado a vigilância das espécies secundárias. Desta forma, pudemos observar no período de cinco anos de pesquisa o índice de unidades domiciliares foi $12 \%$ de infestação e taxa de dispersão triatomínica foi $34 \%$, correspondendo a percentagem das localidades positivas do estado.

Assim, comparando-se os dados anteriores da fauna triatomínica e índices de infecção natural por Trypanosoma cruzi nos municípios de maior risco, verifica-se que as espécies de triatomíneos existentes no Estado de Mato Grosso do Sul são nativas, geralmente se estabelecem no peridomicílio e permanecem ainda com fraca tendência em invadir, ou colonizar o domicílio. Algumas espécies são muito abundantes como o Triatoma sordida e Rhodnius neglectus. Porém apresentam 
índices de infecção natural muito baixos, tal fato parece estar relacionado à associação desses vetores com os ecótopos peridomiciliares ${ }^{15}$.

Quanto ao inquérito sorológico para determinar a prevalência pelo Trypanosoma cruzi em escolares de 7-14 anos em municípios de risco no estado, este foi extremamente baixo. De 2.092 amostras realizadas, entre 1993 a 1995, revelou apenas um sororreagente em Paranhos, município que se localiza na fronteira com o Paraguai Ministério da Saúde ${ }^{15}$ e de 4.151 amostras processadas, entre 1998 a 1999, foram encontrados dois indivíduos infectados ${ }^{21}$.

Concluiu-se que até o momento desta pesquisa, o Estado de Mato Grosso do Sul está livre da transmissão vetorial endêmica da doença de Chagas. Contudo, faz-se necessário manter o serviço de vigilância epidemiológica e entomológica permanente, não só para monitorar uma possível reintrodução do Triatoma infestans no estado, mas também para detectar espécies consideradas nativas, como Triatoma sordida, Panstrongylus megistus, Panstrongylus geniculatus e Rhodnius neglectus que predominam em alguns municípios do Estado e que já demonstraram tendência à domiciliação, devido a ação antrópica que favorece a dispersão e a colonização desses triatomíneos, conseqüentemente, elevando o risco de transmissão vetorial.

\section{AGRADECIMENTOS}

Ao Professor Marcos Gino Fernandes da Universidade da Grande Dourados (UFGD), pela contribuição nas análises estatísticas.

\section{REFERÊNCIAS}

1. Almeida PS, Santos HR, Barata JMS, Obara MT, Ceretti WJr. Ocorrência de Panstrongylus guentheri Berg (Hemiptera: Reduviidae) no Mato Grosso do Sul. Neotropical Entomology 37:107-108, 2008.

2. Carcavallo RU, Girón IG, Jurberg J, Lent H. Bibliographic checklist of the American Triatominae (Hemiptera: Reduviidae). In: Carcavallo RU, Girón IG, Juberg J, Lent H. Atlas dos vetores da Doença de Chagas nas Américas. Fundação Oswaldo Cruz, Rio de Janeiro Vol. I, cap. 4, p. 10-393, 1997.

3. Carcavallo RU, Girón IG, Jurberg J, Lent H. Atlas of Chagas Disease Vectors in the Americas. In: Rodolfo U. Carcavallo, Itamar Galíndez Girón, José Juberg e Herman Lent. Atlas dos vetores da Doença de Chagas nas Américas. Fundação Oswaldo Cruz, Rio de Janeiro,Vol. II, cap.13, p.409-733, 1998.

4. Dias JCP, Loyola CCP, Brener S. Doença de Chagas em Minas Gerais: Situação Atual e Perspectivas. Revista Brasileira de Malariologia e Doenças Tropicais 37:7-28, 1985.

5. Dias JCP, Machado EMM, Borges EC, Moreira EF, Gontijo C, Azevedo BVM. Doença de Chagas em Lassence, Minas Gerais: Reavaliação clínico-epidemiológica 90 anos após a descoberta de Carlos Chagas. Revista da Sociedade Brasileira de Medicina Tropical 35:167-176, 2002.

6. Diotaiuti L. Importância atual e perspectivas de controle de Triatoma sordida em Minas Gerais. Tese de Doutorado, Universidade Federal de Minas Gerais, Belo Horizonte, MG 1991.
7. Forattini OP. Biogeografia, Origem e Distribuição Domiciliação de Triatomíneos no Brasil. Revista de Saúde Pública 14:265-299, 1980.

8. Galvão C, Carcavallo RU, Rocha DS, Jurberg J. A checklist of the current valid species of the subfamily Triatominae Jeannel, 1919 (Hemiptera, Reduviidae) and their geographical distribution, with nomenclatural and taxonomic notes. Zootaxa 202:1-36, 2003. Disponível em www.mapress.com/zootaxa, 2003.

9. Gurgel Gonçalves R, Duarte MA, Ramalho ED, Palma ART, Romaña CA, Cuba Cuba CA. Distribuição espacial de populações de triatomíneos (Hemiptera: Reduviidae) em palmeiras da espécie Mauritia flexuosa no Distrito Federal, Brasil. Revista da Sociedade Brasileira de Medicina Tropical 37:241-247, 2004.

10. Instituto Brasileiro de Geografia e Estatística. População de Mato Grosso do Sul e seus respectivos municípios, segunda estimativa de 2005. Publicado no Diário Oficial União -nr. 168, com data de referência de 31/07/2005. Disponível em http://map.ibge.gov.br, 2005.

11. Lent H, Wygodzinsky P. Revision of the Triatominae (Hemiptera, Reduviidae) and their significance as vectors of Chagas' disease. Bulletin American Museum Natural History 163: 123-520, 1979.

12. Mingoti SA. Análise de dados através de métodos de estatísticas multivariada: uma abordagem aplicada. Editora Universidade Federal de Minas Gerais, Belo Horizonte, 2005.

13. Ministério da Saúde. Relatório do Programa de Erradicação do Triatoma infestans (PETi). Coordenação Regional de Mato Grosso do Sul, Ministério da Saúde, 1992

14. Ministério da Saúde. Controle da Doença de Chagas. Diretrizes Técnicas. Ministério da Saúde, Brasília, 1996.

15. Ministério da Saúde. Proposta para certificação da interrupção da transmissão vetorial da doença de Chagas por Triatoma infestans no Brasil. Ministério da Saúde, Brasília, 2000.

16. Schofield CG, Dias JCP. The Southern Cone initiative against Chagas disease. Advances in Parasitology 42: 1-27, 1998.

17. Silva AR, Rodrigues VLCC, Carvalho ME, CP Junior. Programa de Controle da Doença de Chagas de São Paulo: persistência de alta infestação por triatomíneos em localidades na década de 1990. Rio de Janeiro. Cadernos de Saúde Pública 19:965-971, 2003

18. Silveira AC. Indicadores Operacionais para o Programa de Eliminação de Triatoma infestans. Revista da Sociedade Brasileira de Medicina Tropical 26: 51-54, 1993 .

19. Silveira AC. El control de la enfermedad de Chagas em los Países do Cono Sur da América: História de uma iniciativa internacional. 1991/2001. Uberaba: Revista Sociedade Brasileira de Medicina Tropical, 2002.

20. Silveira AC. Informe de Consultoria. Enfoque de risco na vigilância epidemiológica da doença de Chagas. Organização Pan-americana de Saúde. Mimeo, 2005.

21. Silveira AC, Feitosa VR, Borges R. Distribuição de triatomíneos capturados no ambiente domiciliar, no período de 1975/83, Brasil. Revista Brasileira de Malariologia de Doenças Tropicais 36:15-312, 1984.

22. Silveira AC, Rezende DF. Epidemiologia e Controle da Transmissão Vetorial da Doença de Chagas no Brasil. Revista da Sociedade Brasileira de Medicina Tropical 27: 11-22, 1994.

23. Superintendência de Campanhas de Saúde Pública. Manual de Normas Sobre a Organização e Fundamento de Laboratórios de Diagnóstico da Doença de Chagas, Ministério da Saúde, Brasília, 1981.

24. Silveira Neto S, Nakano O, Barbin D, Villa Nova NA. Manual de ecologia dos insetos, Editora Agronômica Ceres, Piracicaba, 1976

25. Thomazini MJ, Thomazini AP de BW. Diversidade de abelhas (Hymenoptera: Apoidea) em inflorescências de Piper hispidinervum (C.D.C.). Neotropical Entomology 3:27-34, 2002

26. Vinhaes MC, Dias JCP. Doença de Chagas no Brasil. Cadernos de Saúde Pública 16:7-12, 2000. 